\title{
A novel fuzzy logic algorithm for accurate fall detection of smart wristband
}

\author{
Hongtao Zhang ${ }^{1,2}{ }_{(D}$, Muhannand Alrifaai $^{1}$, Keming Zhou ${ }^{2}$ and \\ Huosheng Hu'
}

\begin{abstract}
Falling is a major cause of serious injury or even death for the elderly population. To improve the safety of elderly people, a wide range of wearable fall detection devices have been developed over recent years, such as smart watches, waistbands and other wearable fall detectors. However, most of these fall detection devices are threshold-based and have a high rate of false alarm. This paper presents a novel fuzzy logic fall detection algorithm used in smart wristbands to reduce false alarms and achieve accurate fall detection. Experiments have been conducted in our laboratory and the results show that the proposed algorithm can accurately distinguish fall events from non-fall daily activities such as walking, jumping, clapping, and so forth. It shows good potential for commercial applications.
\end{abstract}

\section{Keywords}

Fall detection, smart wristband, fuzzy system, healthcare system, gyroscope, threshold

\section{Introduction}

As reported, about one third of elderly people over 65 years old have at least one fall per year, which was one of the major health risks leading to the causes of deaths and injuries in the elderly population (Noury et al., 2007). For fall-related injuries, the association between the mortality rate and the time waiting for help has shown a positive correlation (Gurley et al., 1996; Wild et al., 1981). Consequently, elderly people living alone were found to be more seriously hurt than those who were helped within 12 hours (Gurley et al., 1996). In addition to physical injuries, experiencing a fall may lead to a fear of fall, which in turn can bring about the loss of selfconfidence and limitation of activities of daily living (ADLs) (Igual et al., 2013). Detecting falls accurately in time can reduce the severe consequences and is thus of great importance to improve the life quality for elderly people.

The development of automatic fall detection technology has been conducted since the 1990s (Lord and Colvin, 1991). Depending on the type of sensors and devices being used, automatic fall detection technology can be broadly categorized into two groups: (i) non-wearable sensors-based approaches (camera or other ambient sensors) (Er and Tan, 2018; Yang et al., 2015) and (ii) wearable sensors-based approaches (inertial measurement unit (IMU) or other embedded sensors) (Chen et al., 2017; Sucerquia et al., 2018). Although non-wearable sensor-based fall detection systems can provide sufficient information for subject monitoring, it is limited by environmental conditions and coverage areas (Hijaz et al., 2010). For elderly individuals, using wearable devices is a more practical and cost-effective solution to support their independence and safety in terms of mobility and ease of installation.

For wearable fall detection devices, the most commonly used sensors are tri-axial accelerometers or gyroscopes, or a combination of both. Other sensors such as tilt switches, pressure sensors and microphones are used to enhance the fall detection algorithms (Bianchi et al., 2010). The sensors are placed on different places such as head, neck, chest, waist, thigh, wrist, and so forth (Casilari et al., 2017). Several studies have been conducted to search for optimal sensor placement. It is reported that the head, waist and chest are the most effective placement positions, as motions of the human head and trunk are usually minor and these positions are close to the centre of gravity (Delahoz and Labrador, 2014; Kangas et al., 2008; Rucco et al., 2018). However, considering the comfort and convenience, none of the placement positions is favourable and convenient for elderly users to wear on a daily basis (Gjoreski et al., 2016).

A wrist-worn device looks like a regular watch and would be well accepted in almost all situations. It can be small and robust so that it will not drop off easily. It can be waterproof and of low power consumption so that it does not need to be

\footnotetext{
'School of Computer Science and Electronic Engineering, Essex University, UK

${ }^{2}$ August International Ltd, Hoddesdon, UK

\section{Corresponding author:}

Hongtao Zhang, August International Ltd, Rawmec Business Park, Plumpton Road, Hoddesdon ENII OEE, UK.

Email: hzhang@augustint.com
} 
taken off when taking a shower, sleeping or changing clothes. Therefore, wrists are the best wearing positions for elderly people on their daily usages (Quadros et al., 2018). Apart from the elderly, the fall detection function developed in the newly released Apple Watch (Apple Inc. San Francisco California, USA) is available for other aged users. However, the wrist can experience unsteady actions as people use their hands very frequently in the ADLs. There is a lack of research for wrist-worn only fall detectors (Gjoreski et al., 2016).

Degen et al. (2003) developed a prototype of a wrist-worn fall detector, namely Speedy. The algorithm used by Speedy was a typical threshold-based approach that divided a falling process into three steps: high velocity towards the ground, impact on hitting the ground and inactivity on the ground. The accelerometer alone cannot recognize hand motions such as wrist turning or clapping. To reduce false alarms, the gyroscope was introduced by Hsieh et al. (2014). They mounted the devices on the wrists of both hands and used the magnitude of rotation speed as the first threshold for fall detection. They achieved an increased sensitivity of fall detection but compromised the comfort and convenience by using two hands. The usage range was also limited as an additional computer was used to compute the result. A recent wrist-worn fall detector developed by Kukharenko and Romanenko (2017) introduced more thresholds and rules, but only achieved similar accuracy with Degen et al. (2003). By introducing machine learning methods, Quadros et al. (2018) achieved $99.0 \%$ of accuracy for the wrist-worn fall detector; however, the practical application is also limited by using an additional computer to analyse data.

Traditional threshold and rule-based fall detection failed to improve the result, as there is no one line separating fall and non-fall event for the data collected by wrist-worn devices. Instead, the distinguishing features are vaguely defined. In this article, we present a wrist-worn fall detector with a 9-axis motion tracking module, in which a high accuracy and sensitivity fall detection is realized by introducing fuzzy logic algorithm. Both data collection and data computing are conducted on this wristband. In addition, with an external speech recognizer module being integrated, this device can activate or cancel help requests via voice commands. As soon as the help request is activated, or a fall is detected, an SMS with the user's location information will be sent out to a predefined contact number for quick emergency response.

The rest of this paper is organized as follows. Section 2 introduces the system configuration and hardware design of the proposed fall detection wristband. Section 3 details the system software design by describing a novel fall detection algorithm with the fuzzy classification process. Experimental results and test analysis are given in Section 4 to show the feasibility and performance of the proposed algorithm. Finally, a brief conclusion and future work are given in Section 5.

\section{System hardware design}

Figure 1 presents the system configuration of our healthcare system. It contains a healthcare centre in the cloud and smart wristbands for fall detection of individual elderly people. The

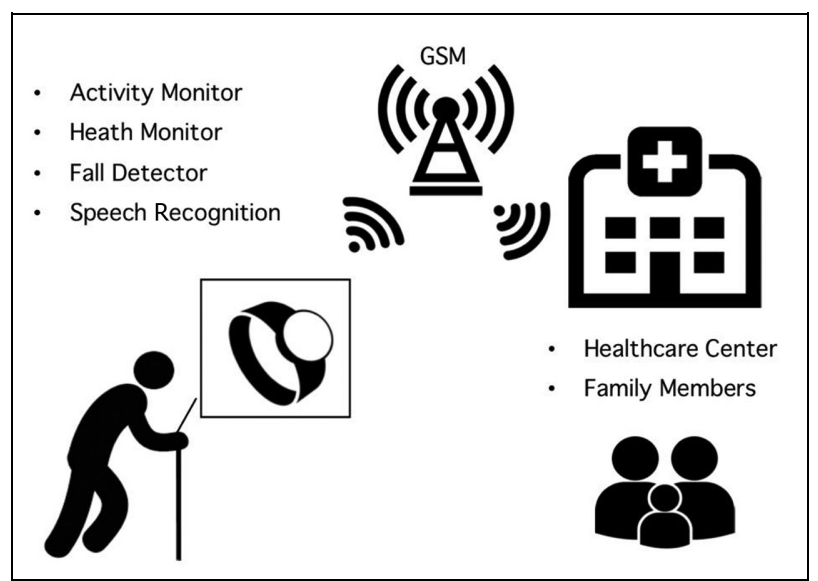

Figure I. The architecture of our healthcare system.

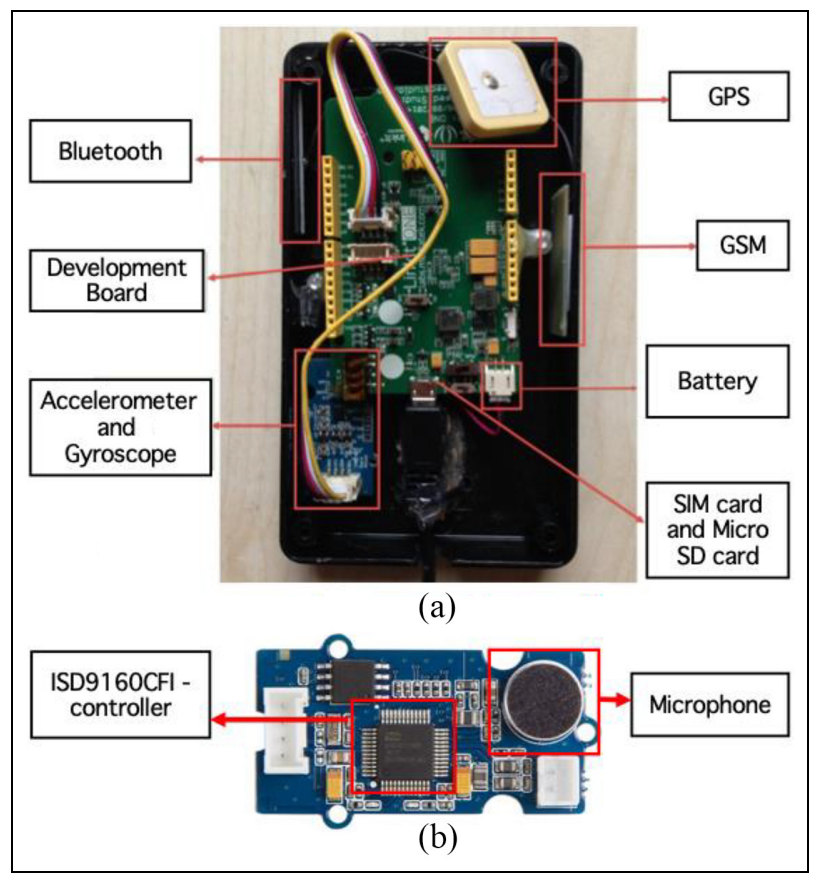

Figure 2. The wristband and speech recognition. (a) The development board. (b) The speech recognizer module.

wristband can constantly record the activity, heartrate and location status of the wearer. In the event of fall, it can send an alarm message with the recorded information to the healthcare centre and/or family members to facilitate an emergency rescue operation. The healthcare centre or family members can check their health status regularly.

Figure 2(a) shows the wristband prototype based on a Linklt ONE development board with built-in Wi-Fi (MT5931), GPS (MT3332) and GSM chipsets, as well as MT2502 that is ultra-small, a low power consumption, and highly integrated ARMv7-based chip with $260 \mathrm{MHz}$ frequency and $4 \mathrm{MB}$ memory, which is excellent for the smart wristband in this project. A 9-axis motion tracking IMU module (Grove 


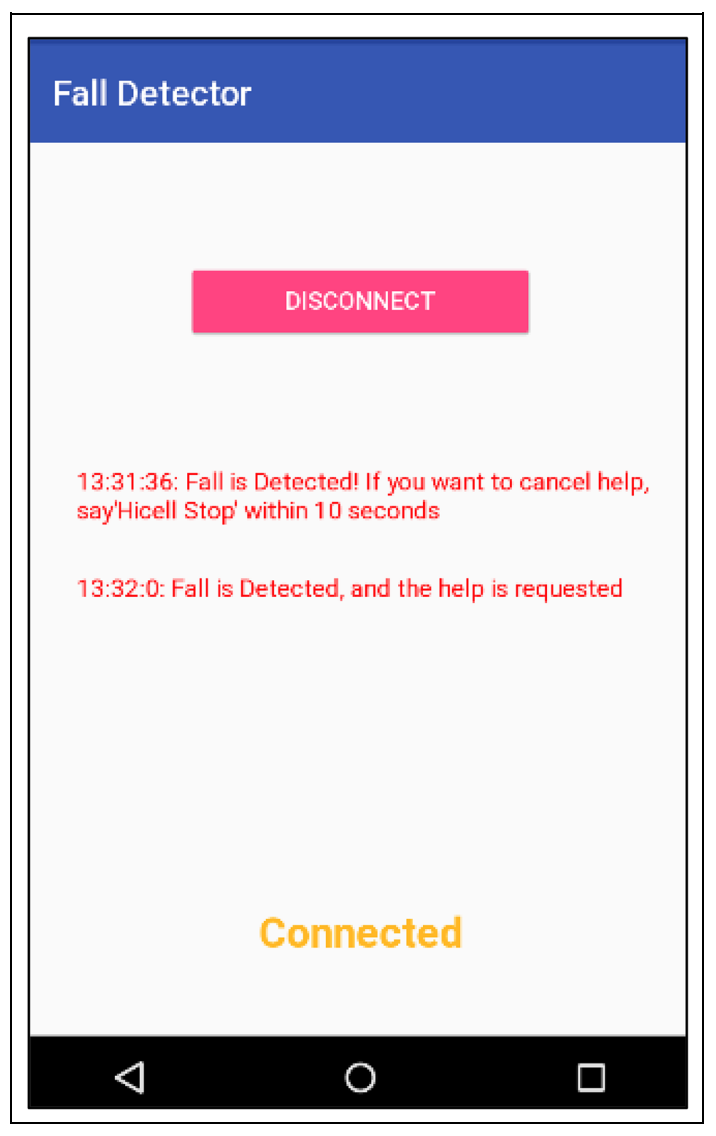

Figure 3. The screenshot of the Android application showing a detected fall in a connected wristband.

- IMU 9DO) is attached by I2C communication protocol with the board. This module is based on MPU-9250 and has a 16bits ADC (Analogue to Digital Converter) for the accelerometer, a 16-bits ADC for the gyroscope and a 16-bits ADC for the magnetometer. In this project, both accelerometer and gyroscope were used to support various ranges of measurement. More specifically, we chose $\pm 2 \mathrm{~g}$ for the accelerometer and \pm 250 degree /second for the gyroscope.

The external speech recognition module we used is "Grove Speech Recognizer", shown in Figure 2(b). This speech recognition module includes a Nuvoton ISD9160, a microphone, a SPI flash, a grove connector, a speaker connector and a LED to enable the device to activate or cancel help request via voice commands. To configure the wristband and get the immediate test result, we used an Android phone to connect the wristband via Bluetooth. A screenshot of the Android application is shown in Figure 3.

\section{System software design}

As the LinkIt One development board supports Arduino Integrated Development Environment (IDE), we can focus on programming the algorithm and not concern ourselves with the connections and drivers. The fall detection program is written in $\mathrm{C} / \mathrm{C}++$ program language, which consists of

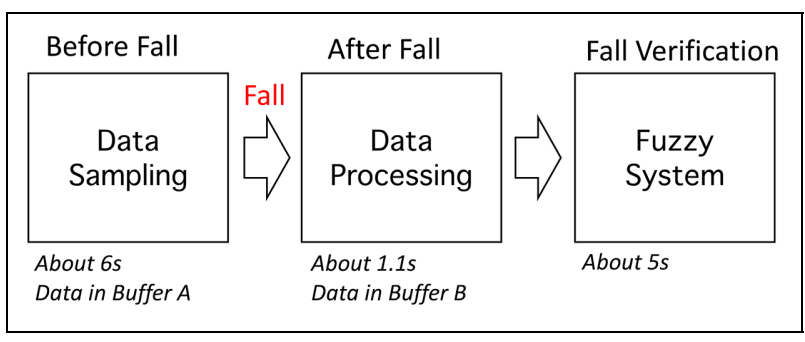

Figure 4. Overview diagram of the data processing.

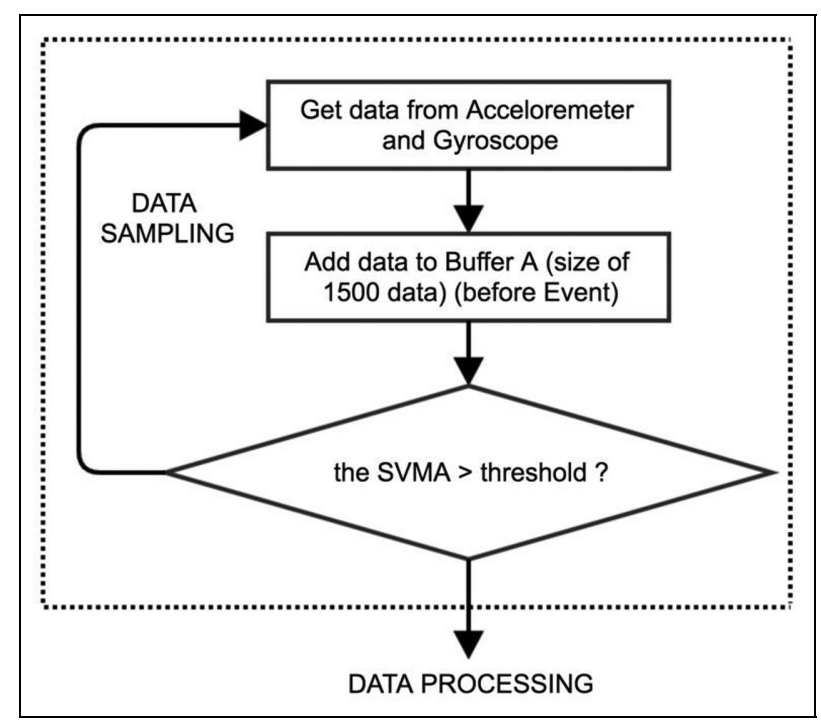

Figure 5. The flowchart of the fall detection algorithm in data sampling stage.

three main stages, namely data sampling, data processing and fuzzy classification.

More specifically, data sampling is continuously storing data from the accelerometer and gyroscope before a fall event. If the sum vector magnitude of the acceleration $\left(\mathrm{SVM}_{\mathrm{A}}\right)$ is over a threshold (threshold-1), the program will proceed to the data processing stage and two fuzz inputs are calculated as two inputs for the followed fuzzy system stage. The first input is the changed angle $(\Delta \mathrm{A})$ of the device during the event, and the second input is the maximum sum vector magnitude of the rotation $\left(\mathrm{SVM}_{\mathrm{G}}\right)$. If the output of the fuzzy system is high (over the threshold-2), the fall will be detected. To avoid false alarm, an inactivity state check (below the threshold-3) is introduced at the end of the fuzzy classification (Figure 4).

\section{Data sampling}

As shown in Figure 5, before proceeding to next stage, we used a buffer to store 3-axis acceleration and 3-axis rotation data from the accelerator and the gyroscope, respectively. The size of the buffer array (Buffer A) is 1500 , before any triggered events. 


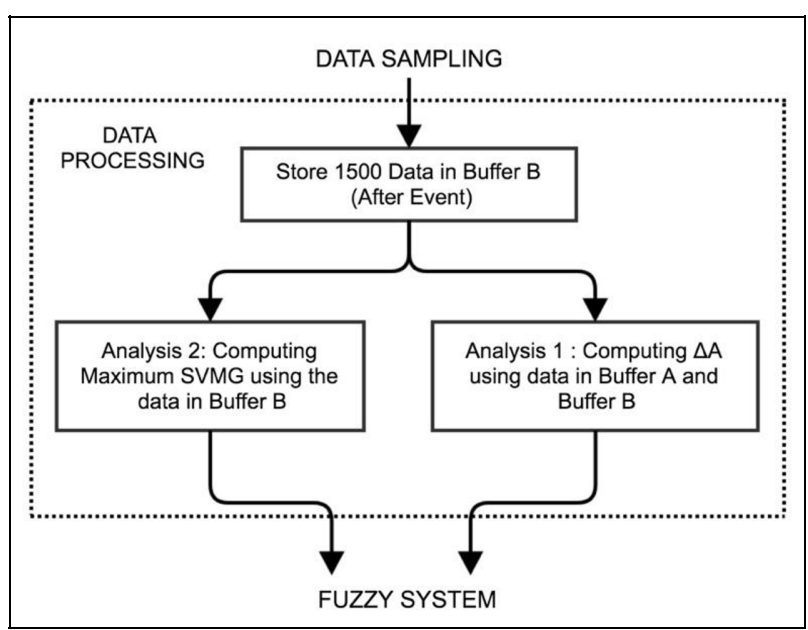

Figure 6. The flowchart of the fall detection algorithm in the data processing stage.

Usually, vertical acceleration is used to detect falls. However, for wrist-worn devices, the acceleration can be in any direction during a fall. In this case, the $\mathrm{SVM}_{\mathrm{A}}$ is calculated as below

$$
S V M_{A}=\sqrt{A_{x}^{2}+A_{y}^{2}+A_{z}^{2}}
$$

where $A_{x}, A_{x}$ and $A_{z}$ represent the acceleration of the $\mathrm{X}, \mathrm{Y}$ and $\mathrm{Z}$ axes, respectively.

According to our experiment results, we used a threshold-1 of $3 \mathrm{~g}$ (Figure 11) to compare the current $\mathrm{SVM}_{\mathrm{A}}$. If the magnitude equals or exceeds the threshold-1, a suspicious event will be detected. Otherwise the program carries on with data storage, $\mathrm{SVM}_{\mathrm{A}}$ computing and threshold-1 comparison.

\section{Data processing}

After the detection of a suspicious event, we used a second buffer array (Buffer B) with the same size (1500) to store data obtained from the accelerometer and gyroscope after the event. Figure 6 shows the flow chart of the program at this stage.

To obtain a general behaviour of the user before the event and more details after the event, 1500 data stored in the buffer A are taken in approximate 6 seconds, whereas the 1500 data in buffer B collected after an event are taken in about 1.1 seconds.

Analysis I: Angle computing. After 1500 data are stored after the event, the first analysis is carried out by computing the angle $(\Delta \mathrm{A})$ changed during the event. To compute the $\Delta \mathrm{A}$, we need to have start and end points of the accelerometer's XYZ. In our algorithm, the start point is the average of 50 values before 1.5 seconds of the event, which are data in range $(1200$ to 1250 ) before the event. The end point is the average of 50 values after 750 milliseconds (1000 to 1050 index of an array) after the event. The $\Delta \mathrm{A}$ is computed as follows
$\Delta A=\cos ^{-1}\left(\frac{\left(A_{x s} * A_{x E}\right)+\left(A_{y s} * A_{y E}\right)+\left(A_{z s} * A_{z E}\right)}{\sqrt{\left(A_{x S^{2}}+A_{y S^{2}}+A_{z S^{2}}\right) *\left(A_{x E}{ }^{2}+A_{y E}{ }^{2}+A_{y E}^{2}\right)}}\right)$

where $A_{x S}, A_{y S}$ and $A_{z S}$ are averaged accelerations in 3-axis at the start point; $\mathrm{A}_{\mathrm{xE}}, \mathrm{A}_{\mathrm{yE}}$ and $\mathrm{A}_{\mathrm{zS}}$ are averaged accelerations in 3-axis at the endpoint.

Analysis 2: Maximum SVM $_{G}$ computing. As hand or arm swinging and turning are normally involved in falling events, we can use data collected from gyroscope to measure the rotation of the device in 3-axes (degree/second). As the device could rotate in all directions during a fall, the directionless $\mathrm{SVM}_{\mathrm{G}}$ is calculated as follows

$$
S V M_{G}=\sqrt{G_{x}^{2}+G_{y}^{2}+G_{z}^{2}}
$$

where $G_{x}, G_{x}$ and $G_{x}$ represent the rotation speed in the X, Y and $\mathrm{Z}$ axes, respectively. In this analysis, the $\mathrm{SVM}_{\mathrm{G}}$ is computed for all 1500 data after the event and we will get a maximum value of $\mathrm{SVM}_{\mathrm{G}}$ for fuzzy classification.

\section{Fuzzy system}

A typical fall is normally processed in three states: zerogravity state, impact state and inactivity state. Therefore, three features are extracted from the accelerometer and gyroscope: $\mathrm{SVM}_{\mathrm{A}}, \Delta \mathrm{A}$ and $\mathrm{SVM}_{\mathrm{G}}$. Measuring the $\mathrm{SVM}_{\mathrm{A}}$ to detect a sudden change of the acceleration in the zero-gravity and impact sates is the most straightforward method to detect falls (Figure 5). However, for wrist-wearing devices, normal ADLs, such as clapping, may easily trigger false alarms. Compared with the trunk, the swinging and turning of the hands are more significant during a fall. Therefore, the introduction of the $\Delta \mathrm{A}$ and the $\mathrm{SVM}_{\mathrm{G}}$ is of great importance for wrist-worn fall detectors.

Two outputs, $\Delta \mathrm{A}$ and the maximum value of $\mathrm{SVM}_{\mathrm{G}}$ can be obtained from the data processing stage. We may compare these two values to two separate thresholds and use the classic Boolean logic "if ... and ...then ..." to determine a fall. However, it is nearly impossible to find two specific thresholds to differentiate falls from all fall-like ADLs for different people, especially for the nature of hands with frequent movements.

To overcome the sharp boundaries between 0 and 1 (fall and non-fall) that are created by thresholds and classic Boolean logic, a fuzzy logic system is introduced. The fuzzy logic system takes the inputs and goes through a series of steps to produce the output based on vagueness and imprecise information. As shown in Figure 7, a typical fuzzy logic operation is processed in three steps:

- Fuzzify all input values into fuzzy membership functions.

- Execute all applicable rules to compute the fuzzy output functions. 


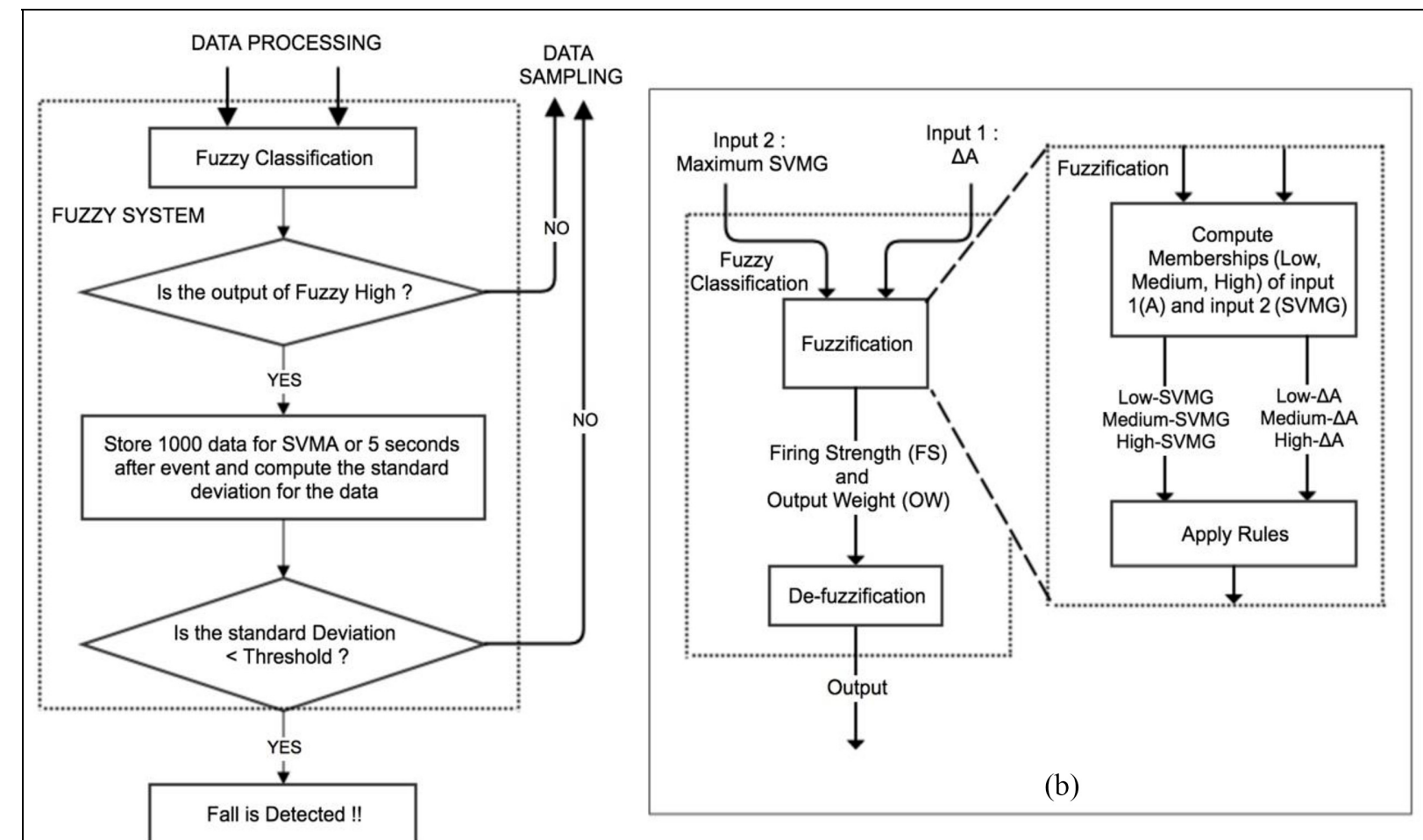

(a)

Figure 7. The flowcharts of the fall detection algorithm in fuzzy system stage. (a) The flowchart of our Fuzzy system. (b) The flowchart of the fuzzy classification in our fuzzy system.

- De-fuzzify the fuzzy output functions to get "crisp" output values.

Fuzzification. Fuzzification is converting inputs to a fuzzy input (Low, Medium and High) with degree of truth. There are two inputs to the systems: $\Delta \mathrm{A}$ and the maximum value of $\mathrm{SVM}_{\mathrm{G}}$. Each input has three memberships: (Low $\Delta \mathrm{A}$, Medium $\Delta \mathrm{A}$, High $\Delta \mathrm{A})$ or $\left(\right.$ Low $\mathrm{SVM}_{\mathrm{G}}, \mathrm{SVM}_{\mathrm{G}}$ and High $\mathrm{SVM}_{\mathrm{G}}$ ), respectively. We select regular polygons shape such as trapezoid and triangle. As shown in Figure 8, the memberships are drawn in a plan, and each membership is bounded with specific values as turning points.

Three turning points $20^{\circ}, 45^{\circ}, 90^{\circ}$ are chosen for the membership shapes of $\Delta \mathrm{A}$. Falls normally happen in $90^{\circ}$, but some falls may happen in an angle less than $90^{\circ}$ and the wrist-worn device cannot measure an accurate $\Delta \mathrm{A}$. Therefore, we put a minimum angle that a fall can happen at $45^{\circ}$ and consider it as the medium angle. The low angle and the high angle are stetted with $20^{\circ}$ and $90^{\circ}$, respectively. The range of all memberships from $0^{\circ}$ to $180^{\circ}$ is determined by the range of minimum and maximum angles that can be computed by the used sensor. Based on that, the event will rather be considered as a fall if the angle is larger than $45^{\circ}$.

Figure 8(b) shows the memberships of the second input to the fuzzy system, maximum $\mathrm{SVM}_{\mathrm{G}}$. It is noted that the range

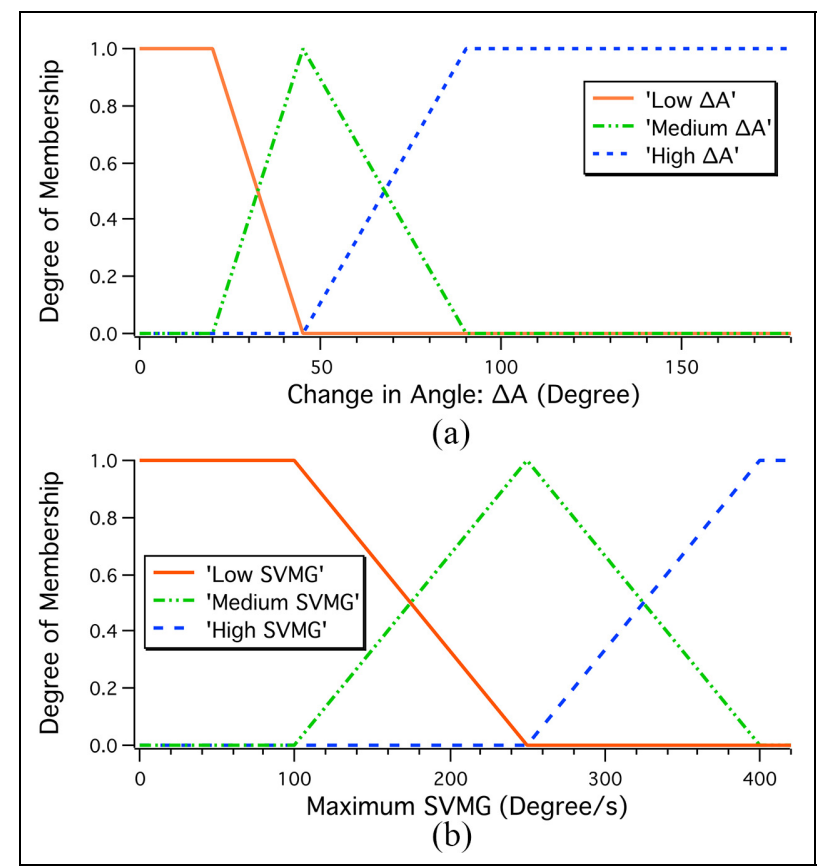

Figure 8. Memberships for inputs: (a) Change in angle: $\Delta \mathrm{A}$. (b) Maximum $\mathrm{SVM}_{\mathrm{G}}$. 
Table I. Rules for fuzzy logic system.

\begin{tabular}{llllll}
\hline Rules & Input I: $\Delta \mathrm{A}$ & Input 2: SVM & Output & Output weight (OW) & Firing strength (FS) \\
\hline I & LOW & LOW & LOW & 10 & FSI \\
2 & LOW & MEDIUM & LOW & 10 & FS2 \\
3 & LOW & HIGH & LOW & 10 & FS3 \\
4 & MEDIUM & LOW & LOW & 10 & FS4 \\
5 & MEDIUM & MEDIUM & MEDIUM & 30 & FS5 \\
6 & MEDIUM & HIGH & HIGH & 50 & FS6 \\
7 & HIGH & LOW & LOW & 10 & FS7 \\
8 & HIGH & MEDIUM & MEDIUM & 30 & FS8 \\
9 & HIGH & HIGH & HIGH & 50 & FS9 \\
\hline
\end{tabular}

of values is within 0 to 433 (degree/second), and the turning points are chosen based on our experimental test to balance sensitivity and specificity. The low magnitude is assigned to 100 , the medium magnitude is assigned to 250 and the maximum one is assigned to 400 . Based on these, an event will be considered as a fall if it has a high magnitude value.

Rules and firing strength. The fuzzy logic systems use rules to process the inputs and produce an output. The rules are linguistic and can be easily recognized and understood by human beings. Therefore, they have been widely deployed in many real-world applications.

After the fuzzification step, the firing strength (FS) should be computed for each rule. We use the Mamdani's minimum operation and AND-output rule in our fall detection algorithm (Mamdani and Assilian, 1999). As shown in Table 1, this rule is simple and widely used, which could minimize false alarms. For example, the FS for the fourth rule (FS4) is the minimum of medium degree membership of input 1 (Medium $\Delta \mathrm{A}$ ) and low degree membership of input 2 (Low $\mathrm{SVM}_{\mathrm{G}}$ ). Consequently, after this step, we will get 9 FS values.

De-fuzzification. De-fuzzification is the last process in our fuzzy logic system, which is to convert fuzzy outputs to a single output. As the inputs have three memberships, the output has three memberships that are low, medium and high. Figure 9 shows the memberships of outputs where all memberships have a triangle shape with an overall range from 0 to 60 and the value of the Output Weight (OW) of 10, 30 and 50 have been assigned to Low, Medium and High outputs, respectively.

The weighted average formula used in de-fuzzification is presented below

$$
\text { Output }=\frac{\sum_{i=1}^{9}\left(F S_{i} * O W_{i}\right)}{\sum_{i=1}^{9} F S_{i}}
$$

where FSi represents fire strength for rule $\mathrm{i}$ and OWi represents output weight for rule $i$. There are 9 FS and OW in total.

Note that the output of de-fuzzification in (4) is the output of our fuzzy logic system. If the output is equal or larger than a threshold (threshold-2), the program will progress to the final step: the verification of the fall.

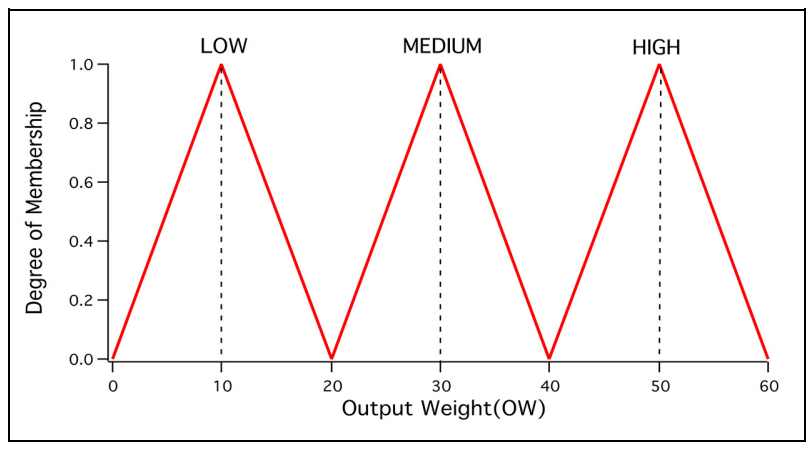

Figure 9. Memberships for output.

Verification of the fall. The output of the fuzzy logic system is sufficient for detecting a fall event. To avoid further false alarms and cover the inactivity-state of the fall, a verification method is added to the algorithm. 1000 data are collected at the inactivity-state for 5 seconds after the impact. A threshold (threshold-3) is set to compare the standard deviation of the $\mathrm{SVM}_{\mathrm{A}}$ of the data. Any result beyond the threshold in the inactivity state will be considered a fall-like ADLs to stop triggering the fall alarm.

\section{SMS notification with localization information}

As soon as a fall is detected, the user can cancel the help request by saying "Stop" within 10 seconds. Otherwise, an SMS with a coordinate GPS location of the person will be sent out. The receiver will receive the SMS with location information of the user, as shown in Figure 10. Moreover, the user can also activate the SMS help request via voice commands whenever it is needed.

\section{System testing and results analysis}

The fall detection experiment was conducted in an indoor environment with a protection of an airbed. In the experiment, four types of fall were tested: forward, backward, right and left. Each type of fall was conducted 13 times with a total of 52 times. To test the performance in ADLs, two young adults wore it all day and experienced no false alarms. More specifically, the daily activities and hand-related activities 


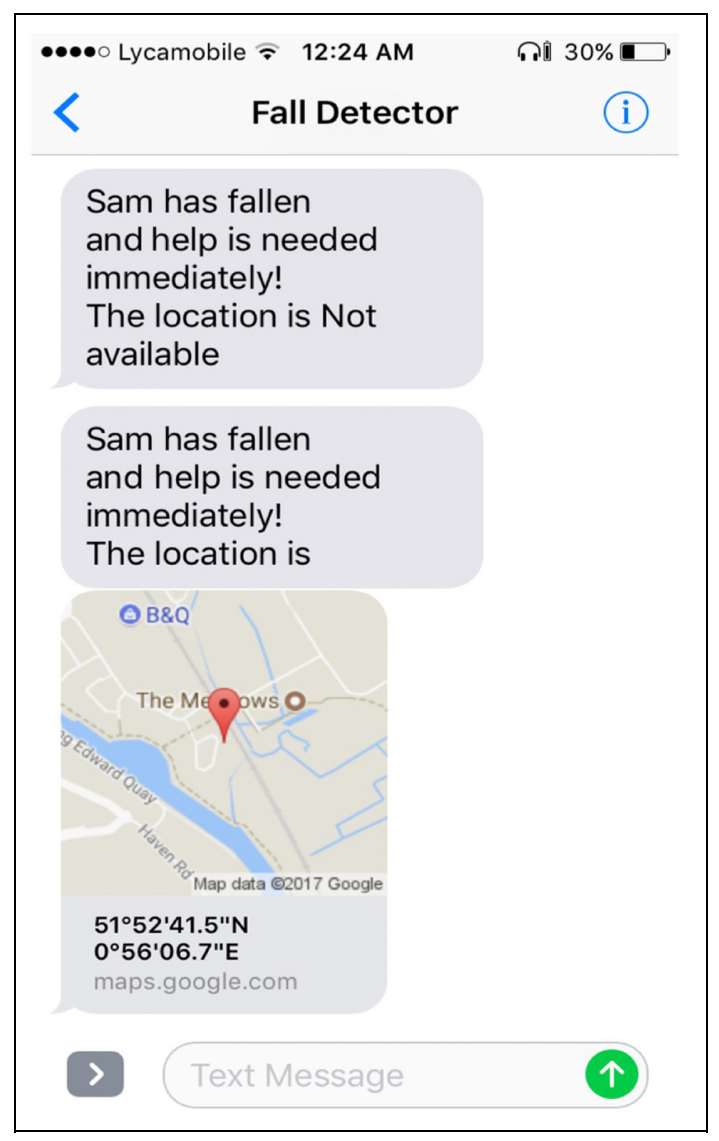

Figure 10. SMS notification received with location information of the wristband user.

Table 2. Fall experiment results.

\begin{tabular}{lllll}
\hline Falling type & $\begin{array}{l}\text { Falling } \\
\text { forward }\end{array}$ & $\begin{array}{l}\text { Falling } \\
\text { backward }\end{array}$ & $\begin{array}{l}\text { Falling } \\
\text { right }\end{array}$ & $\begin{array}{l}\text { Falling } \\
\text { left }\end{array}$ \\
\hline $\begin{array}{l}\text { Detected fall } \\
\text { Missed fall }\end{array}$ & 13 & 13 & 13 & 13 \\
Sensitivity \% & $100 \%$ & 0 & 1 & 1 \\
\hline
\end{tabular}

including sitting down, lying down, walking, running jumping, clapping, wrist turning and arm rotation clapping during jumping were deliberately conducted as well.

Figure 11 shows $\mathrm{SVM}_{\mathrm{A}}$ data for a typical fall and other daily activities. More specifically, the graph on the top is in the event of fall. The middle and bottom graphs show the data when the user is walking and jumping, respectively. Table 2 shows the fall experiment results. All falls were detected by our algorithm except for two cases: one falling right and one falling left. By computing the sensitivity for all types of fall, we got a sensitivity of $100 \%$ for falling forward and backward. In contrast, a sensitivity of $92.3 \%$ for falling right and left was obtained. The sensitivity is used as the ability to detect falls and is defined in the form below

$$
\text { Sensitivity }=\frac{T P}{T P+F N}
$$

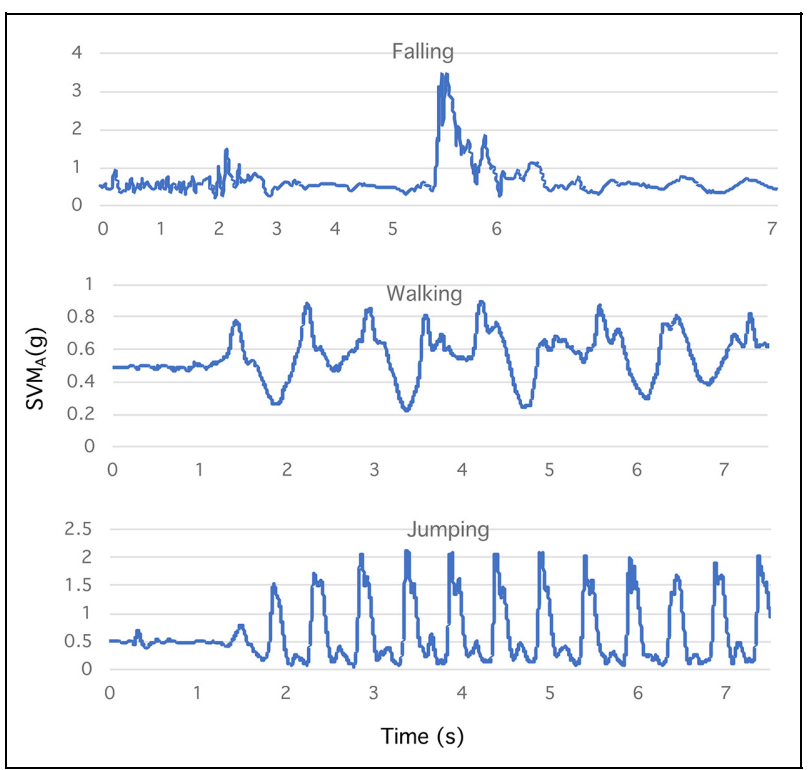

Figure I I. Acceleration data collected by the device. The graph on the top is in the event of fall. The middle and bottom graphs show the data when the user is walking and jumping, respectively.

Table 3. Daily activities experiments - body.

\begin{tabular}{lccccc}
\hline Activity & $\begin{array}{l}\text { Sitting } \\
\text { down }\end{array}$ & $\begin{array}{c}\text { Lying } \\
\text { down }\end{array}$ & Walking & Running & Jumping \\
\hline Times & 5 & 5 & 5 & 5 & 10 \\
False alarm & 0 & 0 & 0 & 0 & 0 \\
Specificity \% & 100 & 100 & 100 & 100 & 100 \\
\hline
\end{tabular}

Table 4. Daily activities experiments - hands.

\begin{tabular}{lllll}
\hline Activity & Clapping & $\begin{array}{l}\text { Wrist } \\
\text { turning }\end{array}$ & $\begin{array}{l}\text { Arm } \\
\text { rotation }\end{array}$ & $\begin{array}{l}\text { Clapping while } \\
\text { jumping }\end{array}$ \\
\hline Times & 10 & 10 & 10 & 10 \\
False alarm & 0 & 0 & 0 & 0 \\
Specificity \% & 100 & 100 & 100 & 100 \\
\hline
\end{tabular}

where TP (Ture Positive) represents the number of falls been correctly detected, FN (False Negative) represents the number of times the system missed detecting a fall.

Table 3 shows the result of conducting daily activities such as sitting down, lying down, walking, running and jumping. Each activity was conducted five times, except for jumping that was conducted 10 times. Consequently, the device did not consider any of them as a fall. Here, we used specificity to measure the system's ability to separate non-fall daily activities from fall events. The specificity is defined in the form below

$$
\text { Specificity }=\frac{T N}{F P+T N}
$$


Table 5. Comparation of sensitivity, specificity and accuracy for wrist-worn fall detectors.

\begin{tabular}{lllllll}
\hline & Degen et al., 2003 & $\begin{array}{l}\text { Hsieh et al., 2014 } \\
\text { (Two hands) }\end{array}$ & $\begin{array}{l}\text { Kukharenko and } \\
\text { Romanenko, 2017 }\end{array}$ & Quadros et al., 2018 & Our result \\
& NA & $95 \%$ & NA & $95.80 \%$ & $100 \%$ & $95.10 \%$ \\
Sensitivity & NA & $96.7 \%$ & NA & $86.50 \%$ & $97.90 \%$ & $100 \%$ \\
Accificity & $65 \%$ & NA & $66 \%$ & $91.10 \%$ & $99.00 \%$ & $98.36 \%$ \\
\hline
\end{tabular}

where TN (True Negative) represents the number of times the system successfully separated the non-fall events from fall events, and FP (False Positive) represents the number of times the system failed to do so.

As elderly people may use their hands frequently, we conducted similar experiments in hand-related activities for our wrist-worn fall detection device. Table 4 shows the results related to hand movements, namely clapping, wrist turning, arm rotation and clapping while jumping. No fall alarm had been triggered in all the none-fall activities and we achieved $100 \%$ specificity for these activities.

Moreover, we can obtain an overall accuracy of about $98.36 \%$ of the fall detection function for all the activities by using the following equation

$$
\text { Accuracy }=\frac{T N+T P}{T P+T N+F P+F N}
$$

An overall $100 \%$ sensitivity and $95.10 \%$ specificity were obtained by using our method. A full result of sensitivity, specificity and accuracy for wrist-worn fall detectors from literatures are listed in Table 5. Ouadros et al. (2018) achieved the best accuracy of about $99 \%$ so far by introducing machine learning approaches. As a fall detector requires instant results from the local device, machine learning with remote computing resources is not a practical solution.

\section{Conclusion and future work}

In this article, we introduced a novel fuzzy logic algorithm for the wrist-worn fall detectors to deal with noisy sensor data and reduce false alarms for their daily operations. Three key features: SVMA, $\Delta$ A and SVMG were abstracted from the IMU in our wristband to represent hand movements during a fall. The originality of this work is the use of fuzzy logic algorithm to deal with noisy sensor data and reduce the false alarm, which has not been deployed in smart wristbands before and can achieve good sensitivity and high accuracy. Compared with fall detectors mounted on the chest or waist where there are less random movements, a wrist-worn device is more comfortable to wear for the target users; for example, elderly people or people with disabilities. All the experimental tests were conducted by young adults and achieved high accuracy and low false positive results.

Our future work will be focused on further tests on the targeted users, that is, elderly people during their daily lives. We will consider more complex falls, such as slow falls and falls with the support of a wall or stick. Finally, we will commercialize our novel fuzzy logic-based fall detection wristbands with power consumption optimisation.

\section{Declaration of conflicting interests}

The author(s) declared no potential conflicts of interest with respect to the research, authorship, and/or publication of this article.

\section{Funding}

The author(s) disclosed receipt of the following financial support for the research, authorship, and/or publication of this article: This research is financially supported by Innovate UK and August International Limited under the Knowledge Transfer Partnership grant (KTP009841). Our thanks also go to Mr Robin Dowling and Mr Ian Dukes for their technical support during the experiment.

\section{ORCID iD}

Hongtao Zhang (D) https://orcid.org/0000-0002-8447-5534

\section{References}

Bianchi F, Redmond SJ, Narayanan MR, et al. (2010) Barometric pressure and triaxial accelerometry-based falls event detection. IEEE Transactions on Neural Systems and Rehabilitation Engineering 18(6): 619-627.

Casilari E, Santoyo-Ramón J-A and Cano-García J-M (2017) Analysis of public datasets for wearable fall detection systems. Sensors (Basel) 17(7): 1513.

Chen K-H, Hsu Y-W, Yang J-J, et al. (2017) Enhanced characterization of an accelerometer-based fall detection algorithm using a repository. Instrumentation Science \& Technology 45(4): 382-391.

Degen T, Jaeckel H, Rufer M, et al. (2003) SPEEDY: A fall detector in a wrist watch. In: Seventh IEEE International Symposium on Wearable Computing, 2003. Proceedings, White Plains, NY, USA, USA, 21-23 October 2003, pp. 184-187. IEEE.

Delahoz YS and Labrador MA (2014) Survey on fall detection and fall prevention using wearable and external sensors. Sensors (Basel) 14(10): 19806-19842.

Er PV and Tan KK (2018) Non-intrusive fall detection monitoring for the elderly based on fuzzy logic. Measurement 124: 91-102.

Gjoreski M, Gjoreski H, Luštrek M, et al. (2016) How accurately can your wrist device recognize daily activities and detect falls? Sensors (Basel) 16(6): 800 .

Gurley RJ, Lum N, Sande M, et al. (1996) Persons found in their homes helpless or dead. New England Journal of Medicine 334(26): 1710-1716. 
Hijaz F, Afzal N, Ahmad T, et al. (2010) Survey of fall detection and daily activity monitoring techniques. In: 2010 International Conference on Information and Emerging Technologies, Karachi, Pakistan, 14-16 June 2010, pp. 1-6. IEEE.

Hsieh S-L, Chen C-C, Wu S-H, et al. (2014) A wrist -worn fall detection system using accelerometers and gyroscopes. In: Proceedings of the 11th IEEE International Conference on Networking, Sensing and Control, Miami, FL, USA, 7-9 April 2014, pp. 518-523. IEEE.

Igual R, Medrano C and Plaza I (2013) Challenges, issues and trends in fall detection systems. Biomedical Engineering Online 12(1): 66.

Kangas M, Konttila A, Lindgren P, et al. (2008) Comparison of lowcomplexity fall detection algorithms for body attached accelerometers. Gait \& Posture 28(2): 285-291.

Kukharenko I and Romanenko V (2017) Picking a human fall detection algorithm for wrist-worn electronic device. In: 2017 IEEE First Ukraine Conference on Electrical and Computer Engineering (UKRCON), Kiev, Ukraine, 29 May-2 June 2017, pp. 276-278. IEEE.

Lord CJ and Colvin DP (1991) Falls in the elderly: Detection and assessment. In: Proceedings of the Annual International Conference of the IEEE Engineering in Medicine and Biology Society Volume 13: 1991, Orlando, FL, USA, USA, 31 October-3 November 1991, pp. 1938-1939. IEEE.
Mamdani EH and Assilian S (1999) An experiment in linguistic synthesis with a fuzzy logic controller. International Journal of HumanComputer Studies 51(2): 135-147.

Noury N, Fleury A, Rumeau P, et al. (2007) Fall detection-principles and methods. In: 2007 29th Annual International Conference of the IEEE Engineering in Medicine and Biology Society, Lyon, France, 22-26 August 2007, pp. 1663-1666. IEEE.

Quadros Td, Lazzaretti AE and Schneider FK (2018) A movement decomposition and machine learning-based fall detection system using wrist wearable device. IEEE Sensors Journal 18(12): 5082-5089.

Rucco R, Sorriso A, Liparoti M, et al. (2018) Type and location of wearable sensors for monitoring falls during static and dynamic tasks in healthy elderly: A review. Sensors (Basel) 18(5): 1613.

Sucerquia A, López J and Vargas-Bonilla J (2018) Real-life/real-time elderly fall detection with a triaxial accelerometer. Sensors (Basel) 18(4): 1101.

Wild D, Nayak U and Isaacs B (1981) How dangerous are falls in old people at home? British Medical Journal (Clin Res Ed) 282(6260): 266-268.

Yang L, Ren YY, Hu H and Tian B (2015) New fast fall detection method based on spatio-temporal context tracking of head by using depth images. Sensors 15(9): 23004-23019. 